\section{$\$$ Research Square}

Preprints are preliminary reports that have not undergone peer review.

They should not be considered conclusive, used to inform clinical practice, or referenced by the media as validated information.

\title{
Multiparametric Magnetic Resonance Imaging in Diagnosis of Small Renal Mass: A Medical Center Experience In Taiwan
}

mingcheng liu ( $\nabla$ penguin2046@gmail.com )

Taichung VGH: Taichung Veterans General Hospital

Ya-Ling Tsou

Taichung VGH: Taichung Veterans General Hospital

Yen-Ting Lin

Taichung VGH: Taichung Veterans General Hospital

Siu-Wan Hung

Taichung VGH: Taichung Veterans General Hospital

Jyh-Wen Chai

Taichung VGH: Taichung Veterans General Hospital

\section{Kun-Yuan Chiu}

Taichung VGH: Taichung Veterans General Hospital

Chih-Hao Chang

Nagoya University: Nagoya Daigaku

Yi-Jui Liu

Feng Chia University https://orcid.org/0000-0001-5865-6836

\section{Research Article}

Keywords: Magnetic resonance imaging (MRI), Apparent diffusion coefficient (ADC), Renal tumor, Renal cell carcinoma (RCC), Angiomyolipoma (AML)

Posted Date: September 1st, 2021

DOI: https://doi.org/10.21203/rs.3.rs-809200/v1

License: () (i) This work is licensed under a Creative Commons Attribution 4.0 International License. Read Full License 


\section{Abstract}

Purpose

Many studies have shown that multiparametric magnetic resonance imaging (MRI) may be helpful for differentiating malignant renal cell carcinomas (RCCs) from benign lesions. However, the key image characteristics between malignancy and benign tumors still require further discussion.

\section{Methods}

We gathered 60 adult patients diagnosed with 72 small renal masses (SRMs) who had received preoperative MRI from 2014 to 2019 at a hospital in Taiwan. The MRI features included conventional MRI parameter, diffusion weighted image (DWI), and dynamic contrast enhanced (DCE) pattern, which are documented and compared among the four common subtype groups: clear cell RCC(ccRCC), papillary RCC(pRCC), angiomyolipoma(AML) and other type RCC. An apparent diffusion coefficient (ADC) value of high and low grade RCCs was also analyzed.

\section{Results}

The results show that $\mathrm{CCRCC}$ had higher T2 weighted signal intensity than the other three subgroups, higher arterial wash-in index (AWI) and ADC value than AML and pRCC, and also manifested a plateau $(n=9,25 \%)$ or washout $(n=27,75 \%)$ dynamic pattern. AMLs exhibited more intravoxel fat than the other three subtypes groups and half of AMLs ( 6 in 12) contained bulk fat. The pRCC demonstrated a more progressive $(n=3,60 \%)$ dynamic pattern than other three subgroups. The ADC value of high-grade RCCs was significantly lower than the low-grade RCCs.

\section{Conclusion}

This may indicate that multiparametric MRI is useful in differentiating those four common pathological types of SRMs and the ADC value may be helpful in evaluation of the histological grade of malignancy.

\section{Introduction}

Small renal masses (SRMs), defined as renal tumors less than $4 \mathrm{~cm}$ in diameter, are less likely to cause clinical symptoms such as hematuria, low back pain, or palpable masses [1]. The three most common subtypes of malignant renal cell carcinoma (RCC) are clear cell, papillary, and chromophobe RCCs [2]. Additionally, the Fuhrman classification grading system divides RCCs into either high-grade or low-grade tumors based on histocytology. In clinical practice, the subtype and histopathologic grade should be taken into consideration when predicting the prognosis of RCC.

In recent years, SRMs have increasingly been incidentally discovered through imaging examinations. [3] However, certain benign tumors, such as angiomyolipoma (AML) and oncocytoma, may display some similar imaging characteristics, making them difficult to differentiate from RCC $[1,4,5]$ and thus making clinicians hesitant to remove SRMs surgically or to actively surveil SRMs.

Renal tumor biopsy (RTB) can provide a pathological diagnosis for uncertain SRMs. Nevertheless, biopsy is not a reliable method for tumor grading due to the possible underestimation of a simple specimen [6]. Additionally, RTB is an invasive procedure with possible complications, such as hemorrhage, infection, pneumothorax, and hollow organ perforation. In the diagnosis or selection of treatment for these SRMs, novel imaging modalities are still useful first tools [7].

Computed tomography (CT) and magnetic resonance imaging (MRI) are the first-line noninvasive imaging methods for both the diagnosis and staging of SRMs. MRI is considered a better tool than CT because there is no radiation exposure to the patient, and diffusion images can also provide extra information in patients with poor renal function for whom contrast medium administration is unsuitable [8]. Multiple MRI parameters can show the intratumoral content of SRMs. For example, T2-weighted, T1-weighted, and chemical shift imaging can reveal manifestations such as hemorrhage, protein, macroscopic fat, or microscopic fat within the tumor; the postcontrast series of dynamic contrast-enhanced (DCE) images can demonstrate the enhancement pattern of solid tumors, while diffusion-weighted imaging (DWI) with apparent diffusion coefficient (ADC) values can illustrate the cellular microenvironment of the tumor by assessing the movement restrictions of free water molecules. These imaging features may be useful in differentiating malignant RCC from benign lesions and classifying RCC subtypes and histological grades [1]. 
Multiparametric MRI has been reported to be helpful in differentiating malignant RCCs from benign lesions in several research studies. However, summaries of the diagnostic values of such parameters have seldom been reported. Therefore, we focused on four pathological subtypes (clear cell RCC, papillary RCC, AML, and other types of RCC) and analyzed the MRI sequences reported to be useful in diagnosing high- and low-grade RCCs as well as benign renal tumors in previous studies.

\section{Methods}

\section{Subjects}

This retrospective study was approved by our institutional review board. By reviewing the medical records from a medical center in Taiwan, this retrospective study identified patients who had undergone surgical resection of a renal mass measuring less than $4 \mathrm{~cm}$ from 2014 to 2019 who also had preoperative multiparametric MRI data of the kidney available for analysis. Patients with renal masses larger than $4 \mathrm{~cm}$ who had a pathologic diagnosis of urothelial carcinoma or renal metastasis were not included. Sixtyeight patients with a total of 80 SRMs were initially selected. Six patients with benign renal cysts and 2 with benign inflammatory disease were excluded because our study aimed to analyze tumor masses.

Finally, 60 adult patients with a total of 72 SRMs were included: 58 (80.6\%) with pathologically confirmed RCC, including 38 (52.8\%) clear cell RCCs (ccRCCs), 9 (12.5\%) papillary RCCs (pRCCs), 3 (4.2\%) chromophobe RCCs (chRCCs), and 8 (11.1\%) other RCC type (4 tuberous sclerosis-associated RCCs, 1 unclassified RCC, 1 Xp11 translocation RCC, and 2 acquired cystic disease-associated RCCs). Additionally, there were 14 SRMs (19.4\%) considered benign renal tumors: 12 (16.7\%) AMLs (1 AML with an epithelioid component) and $2(2.8 \%)$ oncocytomas. Due to the small number of chromophobe RCC $(n=3)$ and oncocytoma $(n=2)$ patients, we did not include these two tumor types in our tumor subtype analysis. We divided the SRMs into four subgroups for comparisons: clear cell RCC, papillary RCC, other types of RCC, and AML.

\section{Image acquisition}

All patients with SRMs underwent MRI using both conventional MRI and DWI sequences. DCE MRI was administered if the patient's renal function allowed. MRI was performed with a 1.5-T body scanner using an 18-channel phased-array body coil with the following protocols:

1. Axial T2-weighted half-Fourier acquisition single-shot turbo spin echo (HASTE): repetition time (TR) $=1200 \mathrm{~ms}$, echo time (TE) = $95 \mathrm{~ms}$, flip angle $=160^{\circ}$, field of view $=36 \times 30 \mathrm{~cm}$, matrix $=320 \times 182$, slice thickness $=5 \mathrm{~mm}$, and gap $=0 \mathrm{~mm}$

2. Axial T1-weighted fast spoiled gradient-recalled echo dual-echo (FSPGR-DE): TR $=142 \mathrm{~ms}, \mathrm{TE}=2.38 \mathrm{~ms}$ and $4.76 \mathrm{~ms}$, flip angle $=$ $70^{\circ}$, field of view $=36 \times 30 \mathrm{~cm}$, and matrix $=256 \times 166$

3. Axial DWI: TR $=3300 \mathrm{~ms}$, TE $=65 \mathrm{~ms}$, field of view $=38 \times 31 \mathrm{~cm}$; matrix $=192 \times 125$; number of excitations $(\mathrm{NEX})=3$; bandwidth $=1736 \mathrm{~Hz} /$ pixel, diffusion mode $=3-\mathrm{scan}$ trace, slice thickness $=5 \mathrm{~mm}$, and gap $=0 \mathrm{~mm}$ with b values $=0$ and $800 \mathrm{~s} / \mathrm{mm}^{2}$ [9-11] . DWI was performed by using a single-shot spin-echo echo-planar imaging sequence with a parallel imaging technique and fat saturation.

4. Dynamic MRI was performed in multiple breath-holds (BHs) using axial 3D fat-suppressed T1-weighted volumetric interpolated breath-hold (VIBE) sequences: TR $=4.5 \mathrm{~ms}, \mathrm{TE}=2.2 \mathrm{~ms}$, flip angle $=15^{\circ}$, field of view $=38 \times 38 \mathrm{~cm}$, matrix $=320 \times 192$, slice thickness $=5 \mathrm{~mm}$, and gap $=0 \mathrm{~mm}$

A gadolinium-based contrast medium was used for the dynamic scan at a dose of $0.1 \mathrm{mmol} / \mathrm{kg}$ of body weight as a bolus injection. Corticomedullary and late nephrographic phase images were acquired 30 and 90 seconds after the contrast medium injection, respectively.

\section{Image interpretation and definition of features}

The data of different MRI sequences, including T2 weighted image signal intensity (T2WI SI), intravoxel fat, bulk fat, DCE enhancement pattern, $A W I$, and $A D C$ values, were recorded. The definition of each MRI feature with its respective grading scale is summarized in Table 1. These images were reviewed and measured by a genitourinary radiologist with five years of experience.

A region of interest $(\mathrm{ROI})$ was placed within a portion of the solid area where the minimum $A D C$ value was identified through visual inspection of the ADC map. The necrotic and hemorrhagic regions were identified on conventional MRI scans and were avoided. 


\section{Statistical analysis}

First, we used the Kolmogorov-Smirnov test to evaluate the normality of the continuous variables, including age, tumor size, arterial wash-in index (AWI), and ADC value. These samples were standardized and compared with a standard normal distribution to determine whether the parametric or nonparametric method should be applied for data analysis. One-way ANOVA (parametric method) and the Kruskal-Wallis test (nonparametric method) were performed to compare the parameters of age, tumor size, AWI, and ADC value among the different renal tumor subtypes. A Dunn-Bonferroni post hoc test was also applied to further select pairwise comparisons. The chi-square test was used for the comparison of categorical variables, such as conventional MRI features, sex, and enhancement patterns.

The t-test (parametric method) and Mann-Whitney U test (nonparametric method) were applied to compare the parameters of AWI and $A D C$ values between low-grade and high-grade malignant tumors. A box-and-whisker plot was created to compare the AWI and ADC values of benign renal lesions and RCCs of different histological subtypes.

SPSS 22.0 software was used for data analysis. Both the AWI and ADC value are expressed as the mean \pm SD. The results were considered statistically significant when the $p$ value was $<0.05$.

\section{Results}

\section{Comparisons among different tumor subtypes}

We compared the imaging and basic features of the four subtypes of renal tumors, $c C R C C, A M L, p R C C$, and other types of RCC. There were significant differences among the four groups of tumors in terms of age, AWI, ADC, size, T2-weighted signal intensity, intravoxel fat, bulk fat, and enhancement pattern $(p=0.033, p<0.001, p<0.001, p=0.012, p<0.001, p<0.001, p<0.001$, and $p<0.001$, respectively). There were no significant differences among these four subtypes regarding sex $(p=0.056)$ and grade $(p=$ 0.426). Finally, the pairwise comparisons that showed the significant differences in the above parameters among the groups of tumors are listed in Table 2 and Table 3.

In the comparison of ccRCC and AML, ccRCC exhibited a higher T2-weighted signal intensity (high: $81.6 \%$ vs $16.7 \%$, and low: $5.3 \%$ vs $66.7 \%$ ) ( $p<0.001$ ); less intravoxel fat (present: $10.5 \%$ vs $75.0 \%$, and absent: $89.5 \%$ vs $25.0 \%)(p<0.001)$; less bulk fat (present: $0.0 \%$ vs $50.0 \%$, and absent: $100.0 \%$ vs $50.0 \%)(p<0.001)$; more type 2 and 3 enhancement patterns (type 2 and type $3: 100.0 \%$ vs $83.3 \%)(p=$ $0.023)$; a higher AWI $(260.9 \pm 116.7$ vs $165.4 \pm 56.5)(p=0.015)$; and a higher ADC value $(1.907 \pm 0.336$ vs $1.191 \pm 0.180)(p<0.001)$.

In the comparison of ccRCC and pRCC, ccRCC exhibited a higher T2-weighted signal intensity (high: $81.6 \%$ vs $22.2 \%$, and low: $5.3 \%$ vs $77.8 \%)(p<0.001)$; more type 3 enhancement patterns (type 3: $75.0 \%$ vs $0.0 \%$, and type $1: 0.0 \%$ vs $60.0 \%)(p<0.001)$; a higher AWI $(260.9 \pm 116.7$ vs $30.5 \pm 13.7)(p<0.001)$; and a higher ADC value $(1.907 \pm 0.336$ vs $0.986 \pm 0.202)(p<0.001)$.

In the comparison of $\mathrm{cCRCC}$ and other types of RCC, ccRCC exhibited a higher T2-weighted signal intensity (high: $81.6 \%$ vs $37.5 \%$, and low: $5.3 \%$ vs $37.5 \%)(p=0.026)$ and larger size $(2.8 \pm 1.0$ vs $1.5 \pm 0.6)(p=0.012)$.

In the comparison of AML and pRCC, AML exhibited more intravoxel fat (present: $75.0 \%$ vs $0.0 \%$, and absent: $25.0 \%$ vs $100.0 \%)(p=$ 0.003 ) and more type 3 enhancement patterns (type $3: 83.3 \%$ vs $0.0 \%$, and type $1: 16.7 \%$ vs $60.0 \%)(p=0.010)$.

In the comparison of AML and other types of RCC, AML exhibited more intravoxel fat (present: $75.0 \%$ vs $0.0 \%$, and absent: $25.0 \%$ vs $100.0 \%)(p=0.003)$.

In the comparison of pRCC and other types of RCC, pRCC exhibited more type 1 enhancement patterns (type 3: $0.0 \%$ vs $83.3 \%$, and type 1: $60.0 \%$ vs $0.0 \%)(p=0.023)$ and a lower AWI $(30.5 \pm 13.7$ vs $225.2 \pm 49.8)(p=0.014)$.

Box-and-whisker plots of the AWI and ADC values of cCRCC, pRCC, other types of RCC, and AML are shown in Fig. 1.

Fig. 2, Fig. 3 and Fig. 4 demonstrate the typical image features of the major types of SRMs. Fig. 2 illustrates the MRI images of a Fuhrman grade II clear cell RCC. The images show that the T2-weighted signal intensity of the tumor is higher than that of the normal renal cortex. There is no obvious signal dropout on the fat-suppressed images compared with nonfat-suppressed T2-weighted images, which indicates that there is no detectable bulk fat. Additionally, there is no obvious signal dropout on the opposed-phase images 
compared with in-phase images, which indicates that there is no detectable intravoxel fat; the AWI is 244.16 , with the presence of a plateau pattern and an $A D C$ value $=1.911 \times 10^{-3} \mathrm{~mm}^{2} / \mathrm{s}$.

Fig. 3 illustrates the MRI images of a benign angiomyolipoma. The images show that the T2-weighted signal intensity of the tumor is lower than that of the normal renal cortex. There is a detectable signal dropout on the opposed-phase images compared with that on the in-phase images, which indicates the presence of intravoxel fat. The AWI was 150.00 , with the presence of a washout enhancement pattern and an $A D C$ value $=1.201 \times 10^{-3} \mathrm{~mm}^{2} / \mathrm{s}$.

Fig. 4 illustrates the MRI images of a Fuhrman grade III papillary RCC. The images show that the T2-weighted signal intensity of the tumor is lower than that of the normal renal cortex. There was no bulk or intravoxel fat component detected in fat-suppressed images or opposed-phase sequences. The AWI was 28.10 , with the presence of a progressive enhancement pattern and an $A D C$ value $=0.896 \times$ $10^{-3} \mathrm{~mm}^{2} / \mathrm{s}$.

\section{Tumor grade comparison}

The study also analyzed differences in imaging and basic features between low-grade and high-grade RCCs, and these results are shown in Table 4. Compared with patients with low-grade ccRCCs, those with high-grade ccRCCs were significantly older $(p=0.024)$, and the ADC value was lower for high-grade ccRCCs $(p<0.001)$. Compared with patients with any subtype of RCC (ccRCC, pRCC, chRCC, and other type RCCs) considered low grade, those with high-grade RCCs were also older $(p=0.015)$, and the ADC value was lower for high-grade RCCs $(p=0.001)$. There were no significant differences between low-grade and high-grade ccRCCs or among all subtypes of RCCs in the parameters of sex, AWI, size, T2-weighted signal intensity, intravoxel fat or enhancement pattern.

The box-and-whisker plot of ADC values between low-grade and high-grade ccRCCs and the corresponding data for all subtypes of RCC are shown in Fig. 5.

\section{Discussion}

In our study, we found that ccRCC showed a higher T2-weighted signal intensity than AML, pRCC, and other types of RCC. This result is in agreement with those from both Kay et al. [3] and Roy C et al.[12], suggesting that pRCC typically has a low signal intensity in T2weighted images and that ccRCC mostly displays a higher signal intensity on T2W images than pRCC, chRCC, and lipid-poor AML.

In essence, AMLs are benign tumors, and regular follow-up imaging is recommended over surgical resection. However, all of the AMLs in our study were pathologically diagnosed through surgery because these were all lipid-poor AMLs that were difficult to distinguish from malignant tumors simply through imaging findings. In our study, bulk fat was found only in AMLs (6 in 12 AMLs), while intravoxel fat was found in both AMLs (9 in 12) and ccRCCs (4 in 34). These findings indicate that the presence of microscopic or macroscopic fat is still the key factor in differentiating AMLs from RCCs. A previous study found that the presence of intravoxel fat may indicate that the tumor is $\mathrm{CCRCC}$ rather than another subtype of RCC. [13] Consistently, none of the other subtypes of RCC were found to have an intratumoral microscopic fat component in our study.

On contrast-enhanced images, the AWI is a quantitative parameter, and the DCE pattern is a qualitative parameter. In our research, cCRCC exhibited a higher AWI than both AML and pRCC, which is in agreement with the findings of Sun et al. [2] and Kay et al. [3] in that there is higher contrast enhancement in ccRCCs than in pRCCs. chRCC had an intermediate signal intensity between that of ccRCC and pRCC. Cornelis et al. [14] also showed that the AWI tended to be lower for pRCCs than for lipid-poor AMLs; however, our study did not observe statistically significant differences between these two tumors. All ccRCCs in our study exhibited a plateau- or washout-type enhancement pattern. These results indicate that the enhancement pattern can play some type of role in differentiating among these tumors. However, this is in contrast to Kay et al.[3] who found nonsignificant differences in enhancement pattern among these tumors. Therefore, further studies on the diagnostic value of the DCE pattern may be needed.

Sandrasegaran et al. [11], Zhang et al. [15] and Lassel et al. [16] found that the ADC levels were significantly different between benign and malignant renal tumors. However, simply comparing the differences between benign and malignant renal tumors can easily lead to statistical errors due to the distribution of the common types of tumors in the population. Therefore, we classified each major tumor subtype separately using different characteristics so that the results will reflect a more accurate comparison. In our study, ccRCC exhibited a higher ADC level than both AML and pRCC. This result contradicted the conclusions of some previous articles, which suggested that benign renal tumors, including cysts, had a higher ADC value than RCCs $[9-11,15,16]$. In contrast, similar to in our study, 
Andreas M. Hötker et al. [17] separately measured the ADC values of AML, oncocytoma, ccRCC, pRCC, chRCC, and unclassified RCC and found that $\mathrm{CCRCC}$ had the highest ADC level in comparison to the other types of renal tumors. Nonetheless, larger and ideally prospective studies of different benign and malignant subtypes of SRMs are still needed to determine the exact ADC value threshold for differential diagnosis.

In our study, the ADC values of high-grade cCRCC and any high-grade RCC were significantly lower than those of low-grade ccRCC and any low-grade RCC, indicating that the ADC value is an independent imaging factor associated with tumor grade. Mytsyk et al. [9] observed a difference in the ADC values of different Fuhrman grades of $c c R C C$, with high-grade tumors presenting lower ADC values and low-grade lesions presenting higher ADC values, which is consistent with our study. High-grade malignant tumors have a higher cell density and stronger angiogenic activity than low-grade malignancies, so water diffusion is more restricted. This phenomenon can be seen not only in ccRCC but also in all RCCs.

In our study, the patients with high-grade ccRCC or any high-grade RCC were significantly older than those with low-grade ccRCC or any low-grade RCC. Mukhopadhyay et al. [18] concluded that patient age is an independent prognostic factor of RCCs. Further clarification of the relationships among patient age, Fuhrman nuclear grade, and tumor stage and their association with prognostic factors may be indicated in the future.

There are several limitations in our study. First, CT is still the major presurgical imaging tool for SRMs surveyed in our hospital, and only patients considered difficult to diagnose undergo MRI, which resulted in an insufficient number of MRI cases. Second, most of the RCCs were CCRCC in our study, and the number of other subtypes of RCC was relatively small. This may make it difficult to statistically analyze different subtypes. Third, some patients with poor renal function were not imaged with a contrast agent, limiting the data collection of AWI and DCE values.

In conclusion, multiparametric MRI is useful in differentiating among common pathological types of SRMs. Additionally, ADC values may be helpful in evaluating the histological grade of RCC.

\section{Declarations}

Funding:

No funding was received to assist with the preparation of this manuscript.

Conflicts of interest/Competing interests:

The authors have no conflicts of interest to declare that are relevant to the content of this article.

Availability of data and material:

Data not available due to ethical and legal restrictions

Code availability:

Not applicable

Authors' contributions:

All authors contributed to the study conception and design. Material preparation, data collection and analysis were performed by MingCheng Liu and Ya-Ling Tsou. The first draft of the manuscript was written by Ming-Cheng Liu. Ya-Ling Tsou and all authors commented on previous versions of the manuscript. All authors read and approved the final manuscript.

Ethics approval:

This research was conducted retrospectively from data obtained for clinical purposes. The study was reviewed and approved by the IRB of Veterans General Hospital, approval number: CE21214A.

Consent to participate: 
The informed consent was not required because this study was retrospective review of patient's images and pathological result, and had no harm to patients.

Consent for publication:

Not applicable

\section{References}

1. Vendrami, C. L., Villavicencio, C. P., DeJulio, T. J., Chatterjee, A., Casalino, D. D., Horowitz, J. M. ... Miller, F. H. (2017). Differentiation of Solid Renal Tumors with Multiparametric MR Imaging. RadioGraphics, 37(7), 2026-2042.

https://doi.org/10.1148/rg.2017170039

2. Sun, M. R., Ngo, L., Genega, E. M., Atkins, M. B., Finn, M. E., Rofsky, N. M., \& Pedrosa, I. (2009). Renal cell carcinoma: dynamic contrast-enhanced MR imaging for differentiation of tumor subtypes-correlation with pathologic findings. Radiology, 250(3), 793-802. https://doi.org/10.1148/radiol.2503080995

3. Kay, F. U., Canvasser, N. E., Xi, Y., Pinho, D. F., Costa, D. N., Diaz de Leon, A. ... Pedrosa, I. (2018). Diagnostic Performance and Interreader Agreement of a Standardized MR Imaging Approach in the Prediction of Small Renal Mass Histology. Radiology, 287(2), 543-553. https://doi.org/10.1148/radiol.2018171557

4. Kim, J. K., Kim, S. H., Jang, Y. J., Ahn, H., Kim, C. S., Park, H. ... Cho, K. S. (2006). Renal angiomyolipoma with minimal fat: differentiation from other neoplasms at double-echo chemical shift FLASH MR imaging. Radiology, 239(1), 174-180. https://doi.org/10.1148/radiol.2391050102

5. Finelli, A., Ismaila, N., Bro, B., Durack, J., Eggener, S., Evans, A. ... Russo, P. (2017). Management of Small Renal Masses: American Society of Clinical Oncology Clinical Practice Guideline. Journal of Clinical Oncology, 35(6), 668-680. https://doi.org/10.1200/jco.2016.69.9645

6. Blumenfeld, A. J., Guru, K., Fuchs, G. J., \& Kim, H. L. (2010). Percutaneous biopsy of renal cell carcinoma underestimates nuclear grade. Urology, 76(3), 610-613. https://doi.org/10.1016/j.urology.2009.09.095

7. Oliva, M. R., Glickman, J. N., Zou, K. H., Teo, S. Y., Mortelé, K. J., Rocha, M. S., \& Silverman, S. G. (2009). Renal cell carcinoma: t1 and 2 signal intensity characteristics of papillary and clear cell types correlated with pathology. AJR Am J Roentgenol, 192(6), 1524-1530. https://doi.org/10.2214/ajr.08.1727

8. Sacco, E., Pinto, F., Totaro, A., D'Addessi, A., Racioppi, M., Gulino, G. ... Bassi, P. (2011). Imaging of renal cell carcinoma: state of the art and recent advances. Urol Int, 86(2), 125-139. https://doi.org/10.1159/000322724

9. Mytsyk, Y., Dutka, I., Borys, Y., Komnatska, I., Shatynska-Mytsyk, I., Farooqi, A. A. ... Kruzliak, P. (2017). Renal cell carcinoma: applicability of the apparent coefficient of the diffusion-weighted estimated by MRI for improving their differential diagnosis, histologic subtyping, and differentiation grade. Int Urol Nephrol, 49(2), 215-224. https://doi.org/10.1007/s11255-016-1460-3

10. Razek, A. A., Farouk, A., Mousa, A., \& Nabil, N. (2011). Role of diffusion-weighted magnetic resonance imaging in characterization of renal tumors. J Comput Assist Tomogr, 35(3), 332-336. https://doi.org/10.1097/RCT.0b013e318219fe76

11. Sandrasegaran, K., Sundaram, C. P., Ramaswamy, R., Akisik, F. M., Rydberg, M. P., Lin, C., \& Aisen, A. M. (2010). Usefulness of diffusion-weighted imaging in the evaluation of renal masses. AJR Am J Roentgenol, 194(2), 438-445.

https://doi.org/10.2214/ajr.09.3024

12. Roy, C., Sauer, B., Lindner, V., Lang, H., Saussine, C., \& Jacqmin, D. (2007). MR Imaging of papillary renal neoplasms: potential application for characterization of small renal masses. Eur Radiol, 17(1), 193-200. https://doi.org/10.1007/s00330-006-0271-9

13. Pedrosa, I., Sun, M. R., Spencer, M., Genega, E. M., Olumi, A. F., Dewolf, W. C., \& Rofsky, N. M. (2008). MR imaging of renal masses: correlation with findings at surgery and pathologic analysis. RadioGraphics, 28(4), 985-1003.

https://doi.org/10.1148/rg.284065018

14. Cornelis, F., Tricaud, E., Lasserre, A. S., Petitpierre, F., Bernhard, J. C., Le Bras, Y. ... Grenier, N. (2014). Routinely performed multiparametric magnetic resonance imaging helps to differentiate common subtypes of renal tumours. Eur Radiol, 24(5), 10681080. https://doi.org/10.1007/s00330-014-3107-z

15. Zhang, H., Gan, Q., Wu, Y., Liu, R., Liu, X., Huang, Z. ... Song, B. (2016). Diagnostic performance of diffusion-weighted magnetic resonance imaging in differentiating human renal lesions (benignity or malignancy): a meta-analysis. Abdom Radiol (NY), 41(10), 1997-2010. https://doi.org/10.1007/s00261-016-0790-z

Page $7 / 13$ 
16. Lassel, E. A., Rao, R., Schwenke, C., Schoenberg, S. O., \& Michaely, H. J. (2014). Diffusion-weighted imaging of focal renal lesions: a meta-analysis. Eur Radiol, 24(1), 241-249. https://doi.org/10.1007/s00330-013-3004-x

17. Hötker, A. M., Mazaheri, Y., Wibmer, A., Zheng, J., Moskowitz, C. S., Tickoo, S. K. ... Akin, O. (2016). Use of DWI in the Differentiation of Renal Cortical Tumors. AJR Am J Roentgenol, 206(1), 100-105. https://doi.org/10.2214/ajr.14.13923

18. Mukhopadhyay, S. G., Mukherjee, K., \& Kr Manna, A. (2015). Renal Tumours in Adults with Correlation between Fuhrman Grading and Proliferative Marker. Iran J Pathol, 10(4), 281-289

\section{Tables}

Table 1 Definition of the imaging features and their grading scales

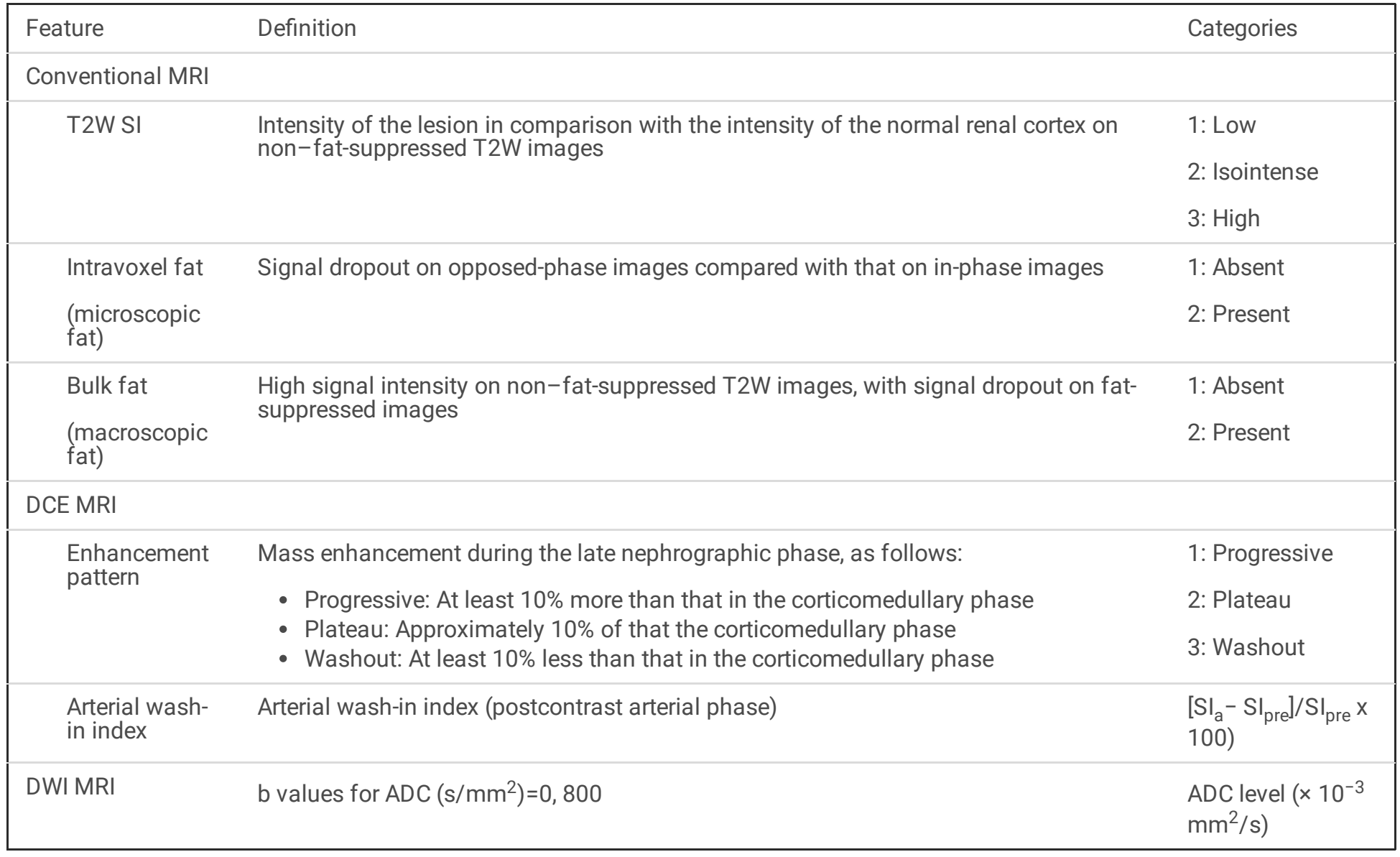

Note. $\mathrm{T} 2 \mathrm{~W} \mathrm{SI}=\mathrm{T} 2$-weighted signal intensity, SI = Signal intensity

Table 2 Comparison of different MRI parameters between each group of tumors (continuous variables) 


\begin{tabular}{|c|c|c|c|c|c|c|c|c|c|}
\hline \multirow[t]{3}{*}{ Group } & & \multirow[t]{3}{*}{$\mathrm{n}$} & \multirow[t]{3}{*}{ Mean } & \multirow[t]{3}{*}{ $\pm S D$} & \multirow{3}{*}{$\begin{array}{l}p \text { value } \\
0.033^{\star}\end{array}$} & \multicolumn{4}{|c|}{ Dunn-Bonferroni test } \\
\hline & & & & & & \multicolumn{4}{|l|}{$p$ value } \\
\hline & Patient age & & & & & & & & \\
\hline 1 & ccRCC & 38 & 50.5 & \pm 16.5 & & 1 vs 2 & 1.000 & 2 vs 3 & 1.000 \\
\hline 2 & AML & 12 & 56.9 & \pm 14.3 & & 1 vs 3 & 0.997 & 2 vs 4 & 0.060 \\
\hline 3 & $\mathrm{pRCC}$ & 9 & 59.1 & \pm 7.3 & & 1 vs 4 & 0.368 & 3 vs 4 & 0.064 \\
\hline \multirow[t]{2}{*}{4} & Other type RCC & 8 & 40.5 & \pm 6.0 & & & & & \\
\hline & AWI & & & & $<0.001 * \star$ & & & & \\
\hline 1 & ccRCC & 36 & 260.9 & \pm 116.7 & & 1 vs 2 & $0.015^{\star}$ & 2 vs 3 & 0.453 \\
\hline 2 & AML & 12 & 165.4 & \pm 56.5 & & 1 vs 3 & $<0.001^{\star \star}$ & 2 vs 4 & 0.436 \\
\hline 3 & $\mathrm{pRCC}$ & 5 & 30.5 & \pm 13.7 & & 1 vs 4 & 1.000 & 3 vs 4 & $0.014^{*}$ \\
\hline \multirow[t]{2}{*}{4} & Other type RCC & 6 & 225.2 & \pm 49.8 & & & & & \\
\hline & $\mathrm{ADC} \times 10^{-3} \mathrm{~mm}^{2} / \mathrm{s}$ & & & & $<0.001^{\star \star}$ & & & & \\
\hline 1 & ccRCC & 38 & 1.907 & \pm 0.336 & & 1 vs 2 & $<0.001^{\star \star}$ & 2 vs 3 & 1.000 \\
\hline 2 & AML & 12 & 1.191 & \pm 0.180 & & 1 vs 3 & $<0.001^{\star \star}$ & 2 vs 4 & 0.423 \\
\hline 3 & $\mathrm{pRCC}$ & 9 & 0.986 & \pm 0.202 & & 1 vs 4 & 0.223 & 3 vs 4 & 0.094 \\
\hline \multirow[t]{2}{*}{4} & Other type RCC & 8 & 1.524 & \pm 0.255 & & & & & \\
\hline & Size & & & & $0.012 *$ & & & & \\
\hline 1 & cCRCC & 38 & 2.8 & \pm 1.0 & & 1 vs 2 & 1.000 & 2 vs 3 & 1.000 \\
\hline 2 & AML & 12 & 2.6 & \pm 1.0 & & 1 vs 3 & 0.594 & 2 vs 4 & 0.111 \\
\hline 3 & $\mathrm{pRCC}$ & 9 & 2.1 & \pm 1.0 & & 1 vs 4 & $0.012^{*}$ & 3 vs 4 & 1.000 \\
\hline 4 & Other type RCC & 8 & 1.5 & \pm 0.6 & & & & & \\
\hline
\end{tabular}

Note. ${ }^{*} p<0.05,{ }^{*} p<0.01$

Table 3 Comparison of different MRI parameters between each group of tumors (categorical variables) 


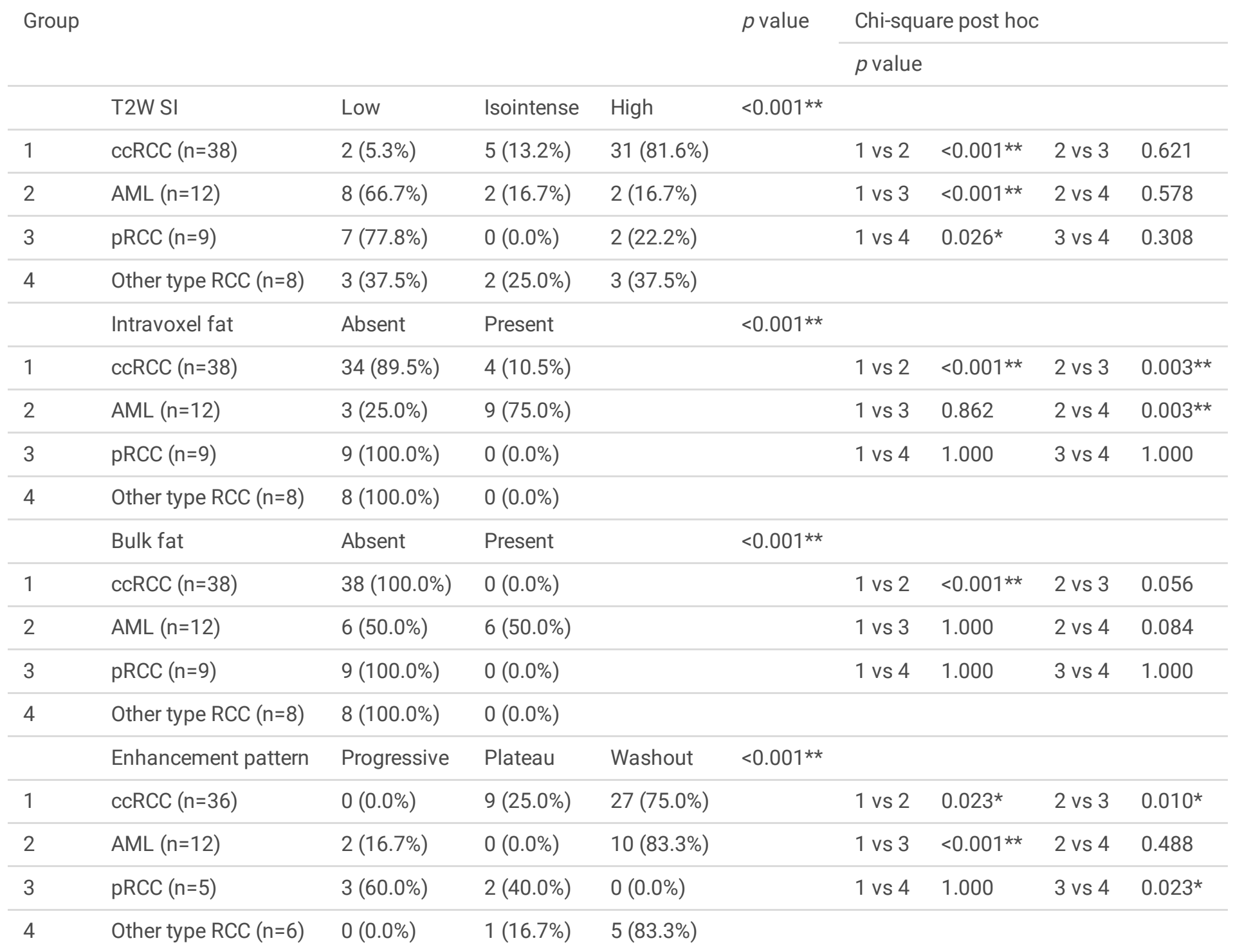

Note. ${ }^{\star} p<0.05,{ }^{* *} p<0.01$

Table 4 Comparison of imaging and basic features between low-grade and high-grade ccRCCs and among all subtypes of RCCs 


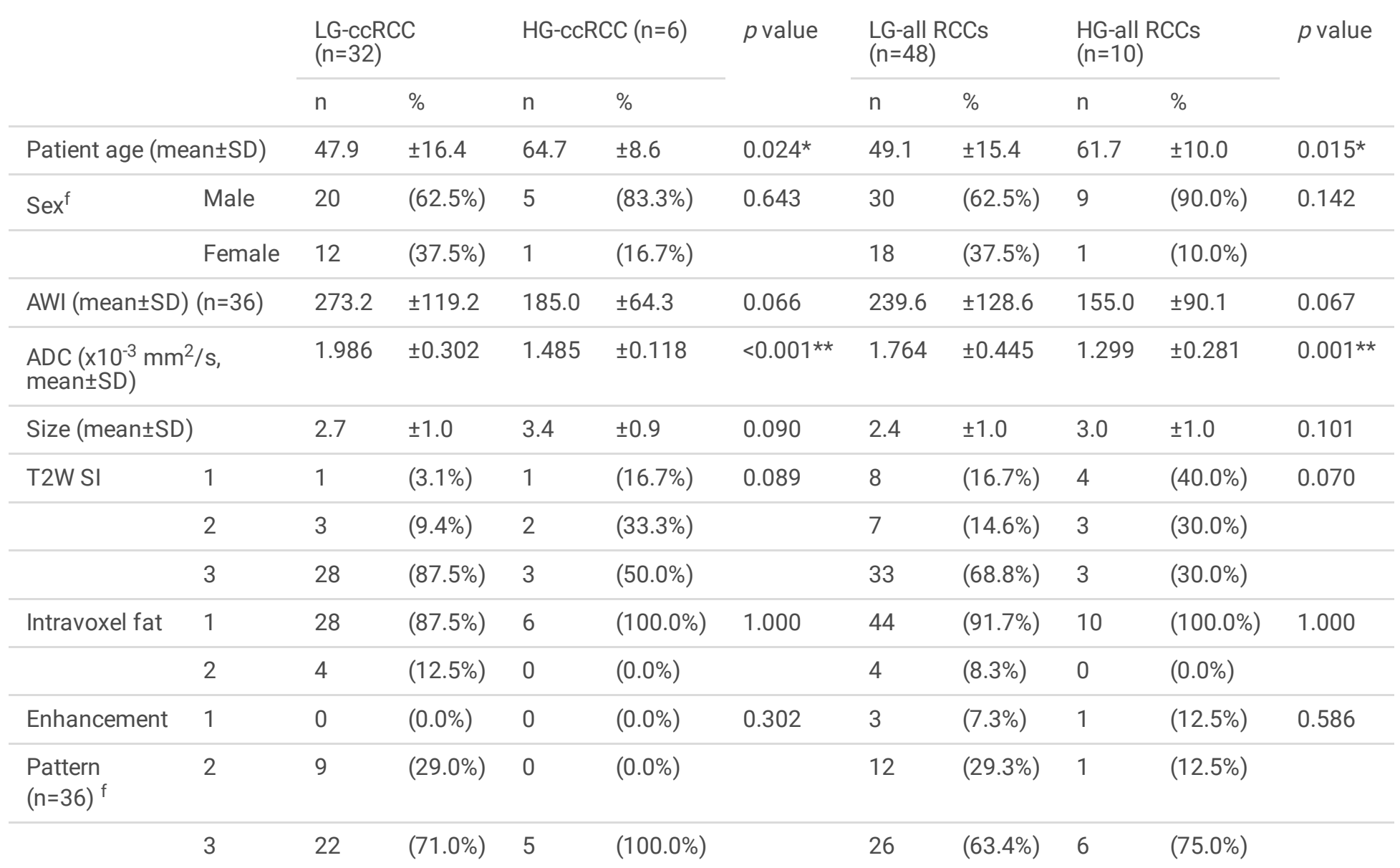

Note. $\mathrm{LG}=$ low-grade, $\mathrm{HG}=$ high-grade, chi-square test. ${ }^{\mathrm{f}}$ Fisher's exact test. Mann-Whitney $\mathrm{U}$ test. ${ }^{\star} p<0.05,{ }^{\star \star} p<0.01$.

\section{Figures}
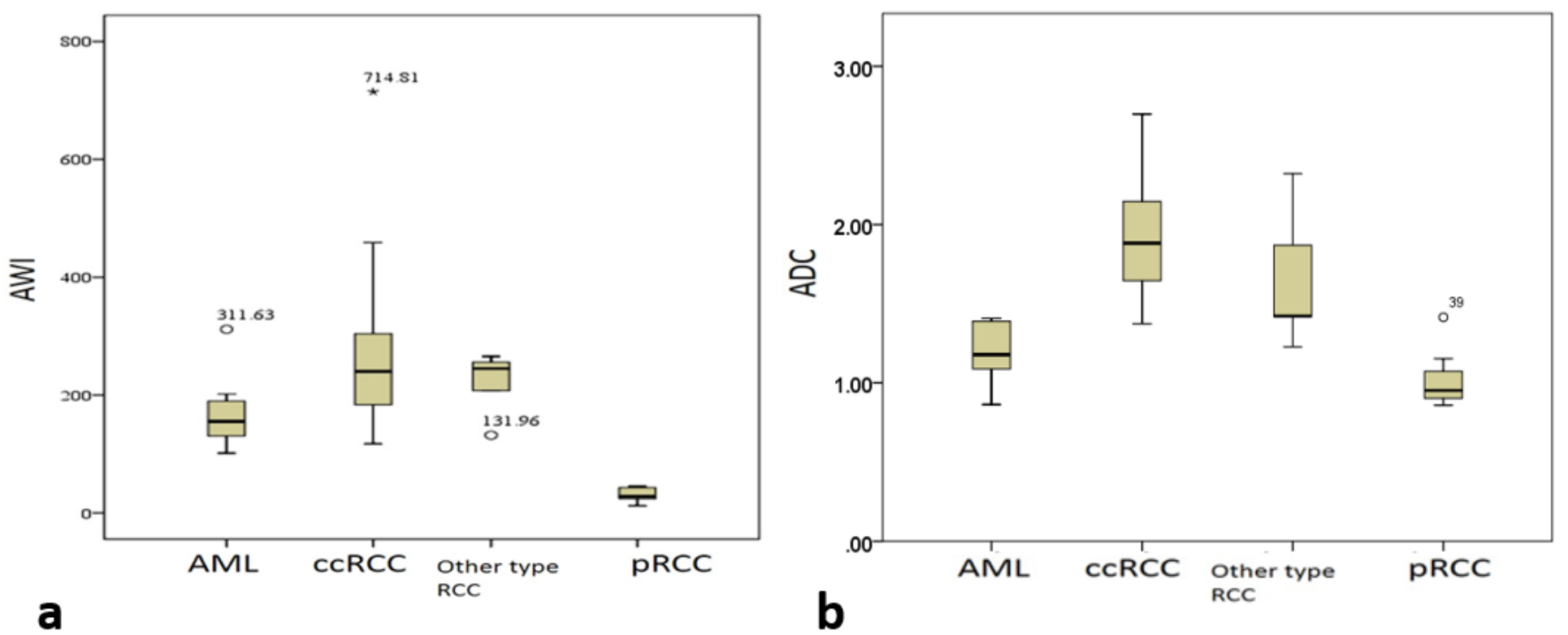

Figure 1

a. Box-and-whisker plot of the AWIs of SRMs; b. Box-and-whisker plot of the ADC values of SRMs (The boxes indicate the interquartile range, the whiskers indicate the range of all values, and the horizontal line within the box indicates the median value, confidence interval (Cl)-95\%) 


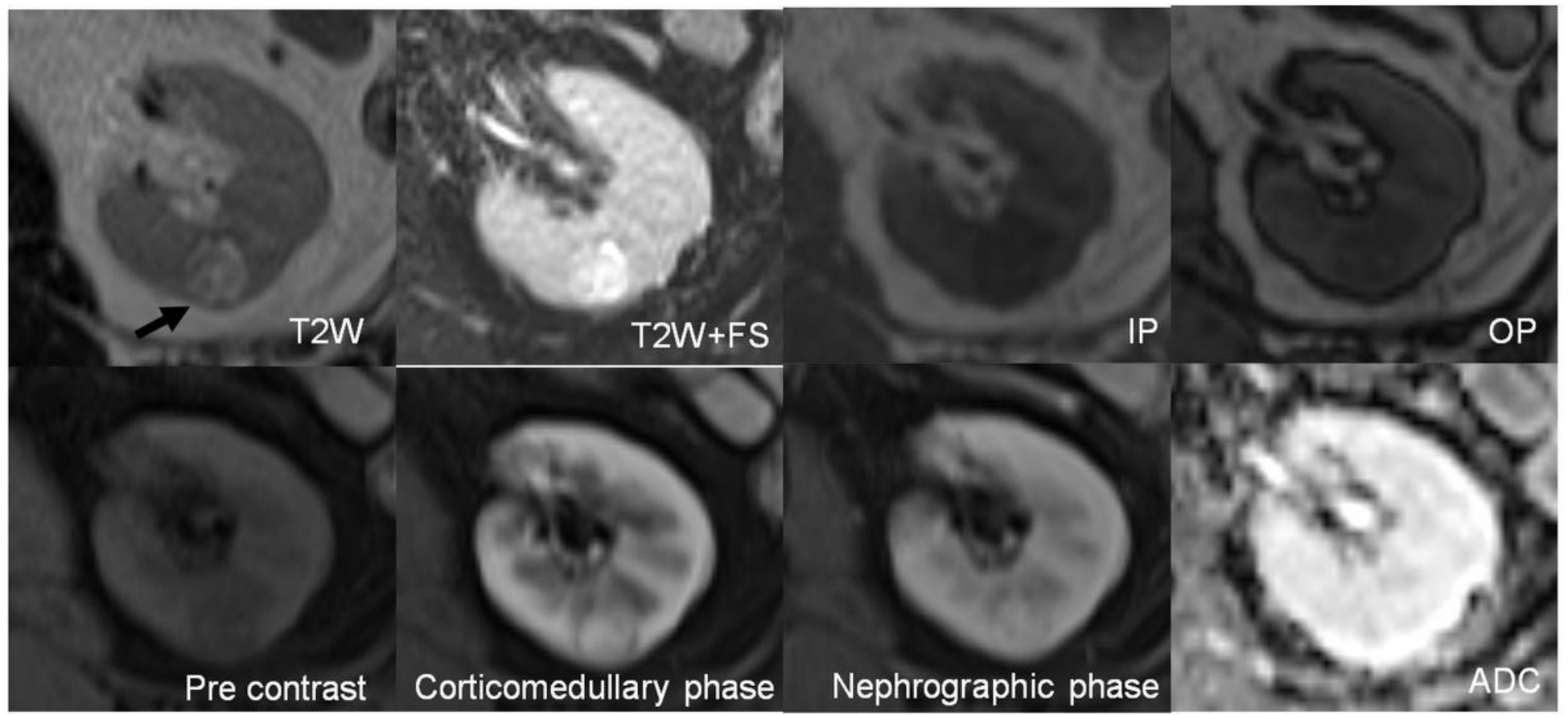

\section{Figure 2}

MRI scans of ccRCC Note. T2W = T2-weighted signal intensity, FS = Fat-suppressed, IP = In-phase images, OP = Opposed-phase images, $A D C=$ Apparent diffusion coefficient

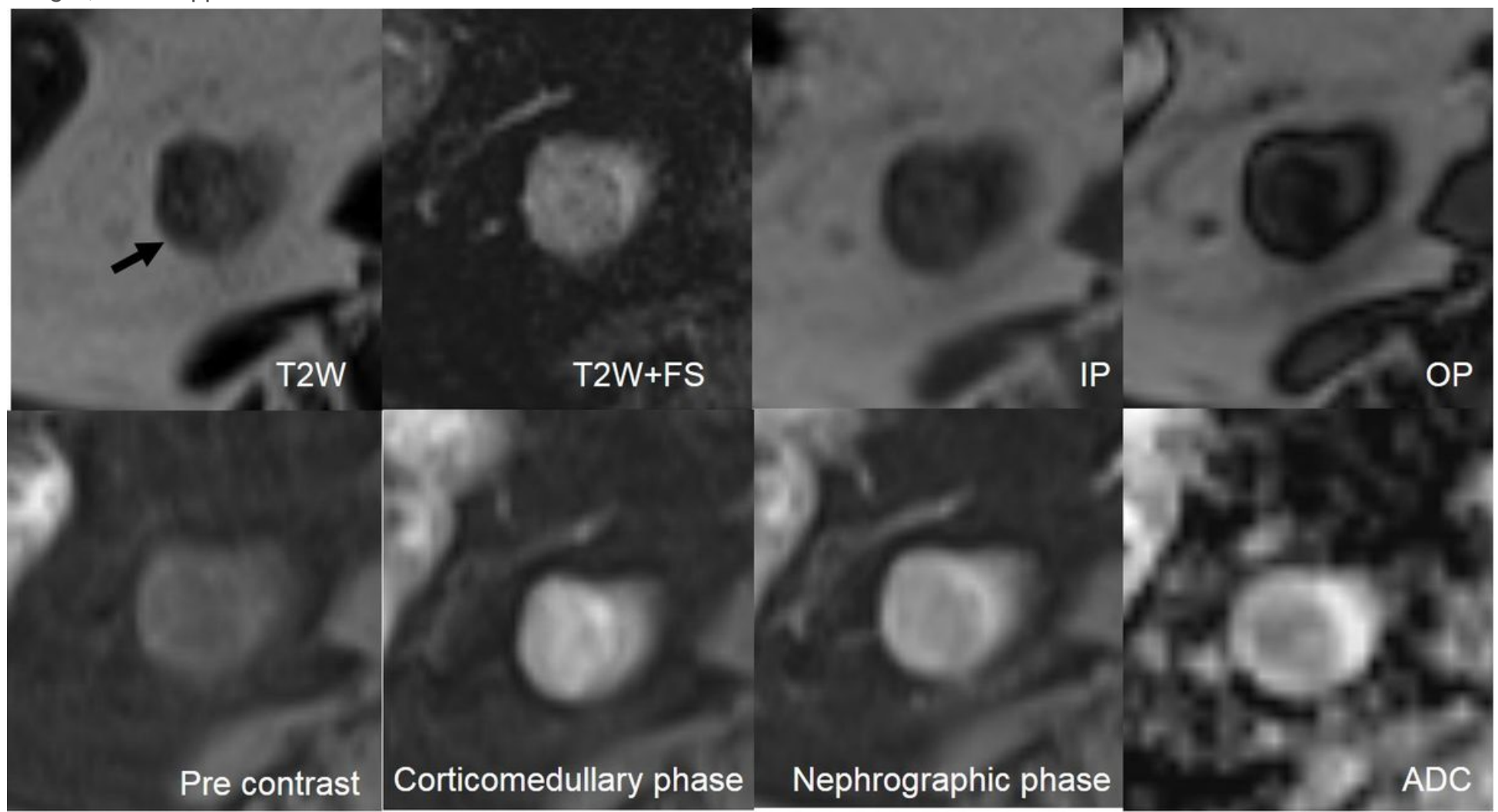

\section{Figure 3}

MRI scans of AML Note. T2W = T2-weighted signal intensity, FS = Fat-suppressed, IP = In-phase images, OP = Opposed-phase images, $A D C=$ Apparent diffusion coefficient 


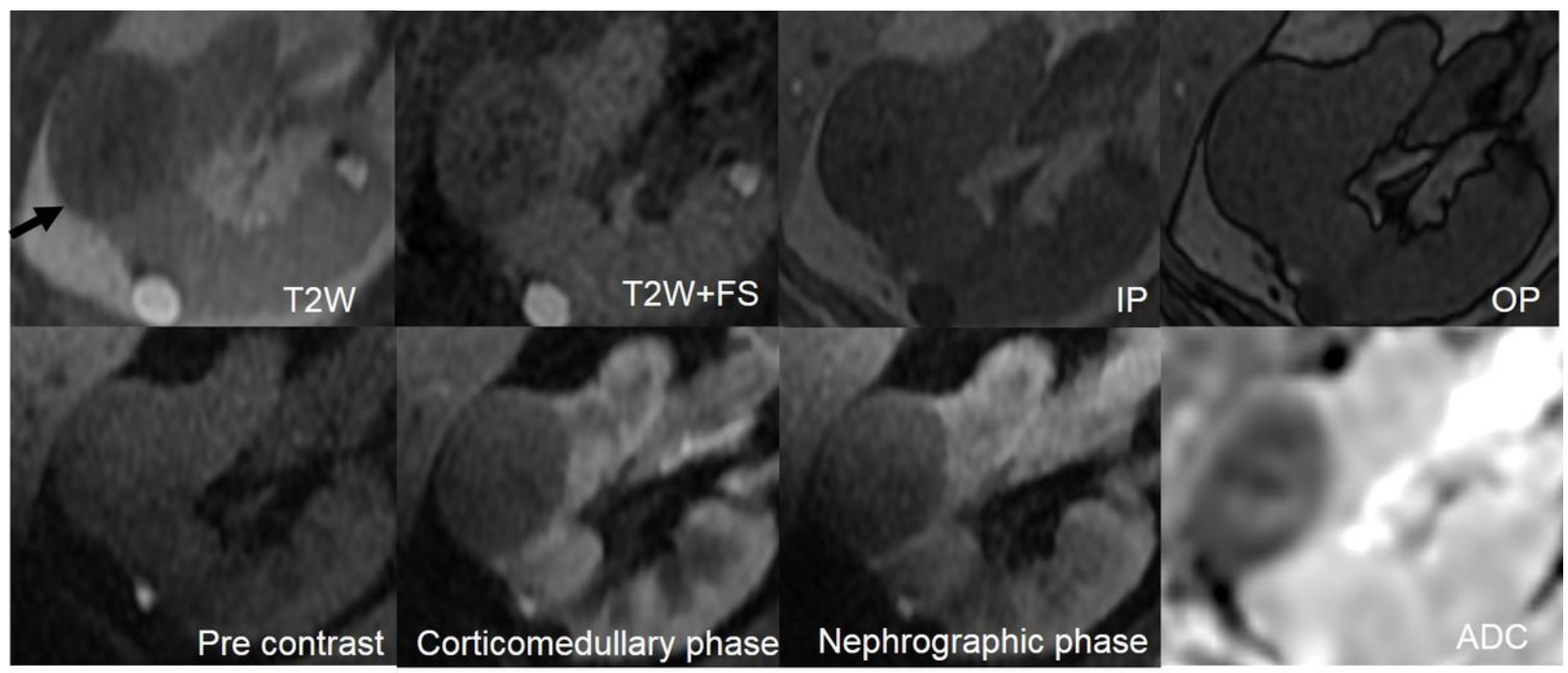

\section{Figure 4}

MRI scans of pRCC Note. T2W = T2-weighted signal intensity, FS = Fat-suppressed, IP = In-phase images, OP = Opposed-phase images, $A D C=$ Apparent diffusion coefficient

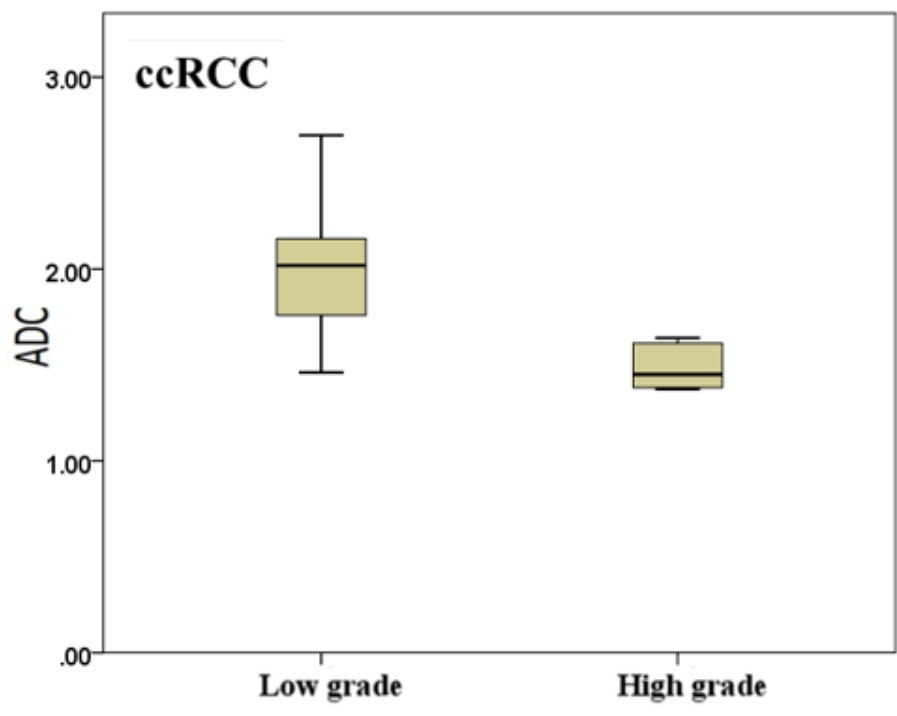

a

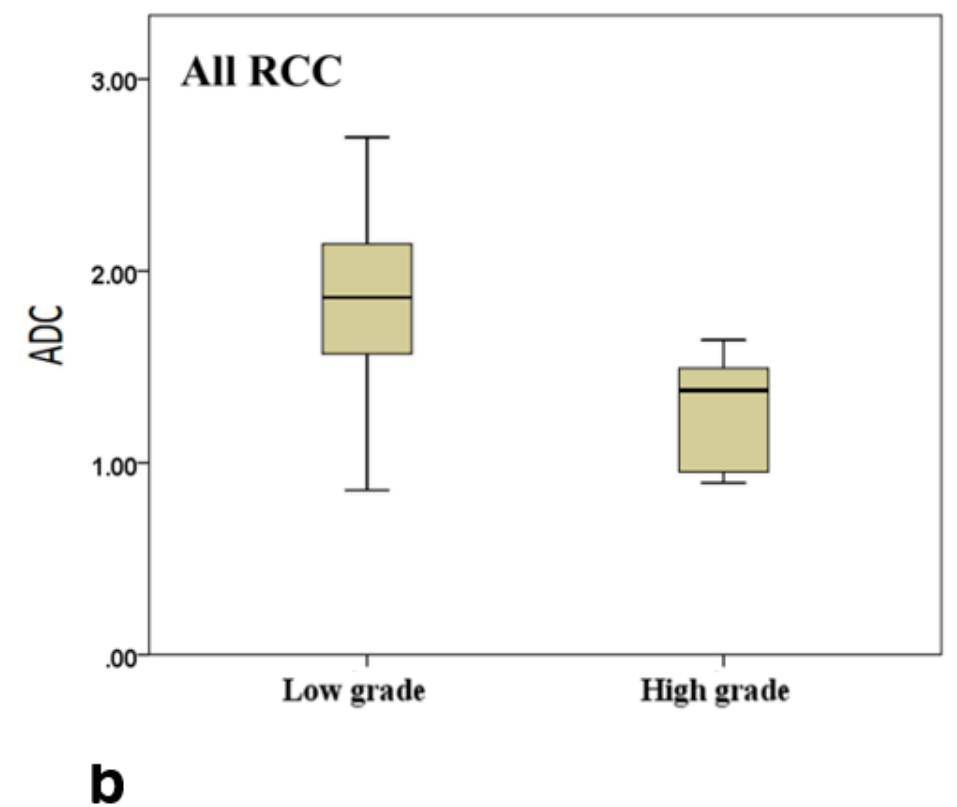

\section{Figure 5}

a. Box-and-whisker plot of the ADC values of low-grade and high-grade ccRCCs; $b$. Box-and-whisker plot of the ADC values of low-grade and high-grade RCCs of any subtype. (The boxes indicate the interquartile range, the whiskers indicate the range of all values, and the horizontal line within the box indicates the median value, confidence interval (CI)-95\%) 